\title{
Bilateral round ligament varicosities mimicking inguinal hernia during pregnancy
}

\author{
F. F. A. IJpma • K. M. Boddeus · H. H. de Haan • \\ D. van Geldere
}

Published online: 25 July 2008

(C) Springer-Verlag 2008

\section{Erratum to: Hernia}

DOI 10.1007/s10029-008-0395-8

The first author's name was spelled the wrong way. It is F.F.A. IJpma instead of F.F.A. Ijpma. 